\title{
Measurement of Spin-Density Matrix Elements for $\phi$-Meson Photoproduction from Protons and Deuterons Near Threshold
}

\author{
W.C. Chang, ${ }^{1}$ D.S. Ahn, ${ }^{2,3}$ J.K. Ahn, ${ }^{3}$ H. Akimune, ${ }^{4}$ Y. Asano,${ }^{5}$ S. Daté,${ }^{6}$ H. Ejiri, ${ }^{2}$ \\ H. Fujimura, ${ }^{7}$ M. Fujiwara, ${ }^{2}{ }^{2}$ S. Fukui,${ }^{9}$ S. Hasegawa, ${ }^{2}$ K. Hicks, ${ }^{10}$ K. Horie,${ }^{2}$ T. Hotta,${ }^{2}$ \\ K. Imai, ${ }^{11}$ T. Ishikawa,${ }^{7}$ T. Iwata, ${ }^{12}$ Y. Kato, ${ }^{2}$ H. Kawai, ${ }^{13}$ K. Kino, ${ }^{2}$ H. Kohri, ${ }^{2}$ \\ N. Kumagai ${ }^{6}$ P.J. Lin, ${ }^{14}$ S. Makino, ${ }^{15}$ T. Matsuda, ${ }^{16}$ T. Matsumura,${ }^{17}$ N. Matsuoka,${ }^{2}$ \\ T. Mibe,${ }^{2}$ M. Miyabe,${ }^{11}$ Y. Miyachi,${ }^{12}$ N. Muramatsu,${ }^{2}$ T. Nakano,${ }^{2}$ M. Niiyama,${ }^{11}$ \\ M. Nomachi, ${ }^{18}$ Y. Ohashi,${ }^{6}$ H. Ohkuma,${ }^{6}$ T. Ooba,${ }^{13}$ D.S. Oshuev,${ }^{1}$ C. Rangacharyulu, ${ }^{19}$
} A. Sakaguchi, ${ }^{18}$ P.M. Shagin, ${ }^{20}$ Y. Shiino,${ }^{13}$ H. Shimizu, ${ }^{7}$ Y. Sugaya,,${ }^{18}$ M. Sumihama,${ }^{2}$ Y. Toi,${ }^{16}$ H. Toyokawa,${ }^{6}$ M. Uchida,${ }^{21}$ A. Wakai, ${ }^{22}$ C.W. Wang, ${ }^{1}$ S.C. Wang, ${ }^{1}$ K. Yonehara, ${ }^{4}$ T. Yorita, ${ }^{2,6}$ M. Yoshimura, ${ }^{23}$ M. Yosoi, ${ }^{2}$ and R.G.T. Zegers ${ }^{24}$

(LEPS Collaboration)

${ }^{1}$ Institute of Physics, Academia Sinica, Taipei 11529, Taiwan

${ }^{2}$ Research Center for Nuclear Physics, Osaka University, Ibaraki, Osaka 567-0047, Japan

${ }^{3}$ Department of Physics, Pusan National University, Busan 609-735, Korea

${ }^{4}$ Department of Physics, Konan University, Kobe, Hyogo 658-8501, Japan

${ }^{5}$ XFEL Project Head Office, RIKEN 1-1, Koto Sayo Hyogo 679-5148, Japan

${ }^{6}$ Japan Synchrotron Radiation Research Institute, Sayo, Hyogo 679-5143, Japan

${ }^{7}$ Laboratory of Nuclear Science, Tohoku University, Sendai, Miyagi 982-0826, Japan

${ }^{8}$ Quantum Beam Science Directorate,

Gamma-ray Nondestructive Assay Research Group,

Japan Atomic Energy Agency, Tokai-mura, Ibaraki 319-1195, Japan

${ }^{9}$ Department of Physics, Nagoya University, Aichi 464-8602, Japan

${ }^{10}$ Department of Physics and Astronomy, Ohio University, Athens, Ohio 45701, USA

${ }^{11}$ Department of Physics, Kyoto University, Kyoto 606-8502, Japan

${ }^{12}$ Department of Physics, Yamagata University, Yamagata 990-8560, Japan

${ }^{13}$ Department of Physics, Chiba University, Chiba 263-8522, Japan

${ }^{14}$ Department of Physics, National Kaohsiung Normal University, Kaohsiung 824, Taiwan 
${ }^{15}$ Wakayama Medical University, Wakayama, 641-8509, Japan

${ }^{16}$ Department of Applied Physics, Miyazaki University, Miyazaki 889-2192, Japan

${ }^{17}$ Department of Applied Physics, National Defense Academy in Japan, Yokosuka, Kanagawa 239-8686, Japan

${ }^{18}$ Department of Physics, Osaka University, Toyonaka, Osaka 560-0043, Japan

${ }^{19}$ Department of Physics and Engineering Physics, University of Saskatchewan, Saskatoon SK S7N 5E2, Canada

${ }^{20}$ School of Physics and Astronomy, University of Minnesota, Minneapolis, Minnesota 55455, USA

${ }^{21}$ Department of Physics, Tokyo Institute of Technology, Tokyo 152-8551, Japan

${ }^{22}$ Akita Research Institute of Brain and Blood Vessels, Akita, 010-0874, Japan

${ }^{23}$ Institute for Protein Research, Osaka University, Suita, Osaka, 565-0871, Japan

${ }^{24}$ National Superconducting Cyclotron Laboratory, Michigan State University, East Lansing, Michigan 48824, USA

\begin{abstract}
The LEPS/SPring-8 experiment made a comprehensive measurement of the spin-density matrix elements for $\gamma p \rightarrow \phi p, \gamma d \rightarrow \phi p n$ and $\gamma d \rightarrow \phi d$ at forward production angles. A linearly polarized photon beam at $E_{\gamma}=1.6-2.4 \mathrm{GeV}$ was used for the production of $\phi$ mesons. The natural-parity Pomeron exchange processes remain dominant near threshold. The unnatural-parity processes of pseudoscalar exchange is visible in the production from the nucleons but is greatly reduced in the coherent production from deuterons. There is no strong $E_{\gamma}$ dependence, but there is some dependence on momentum transfer. A small but finite value of the spin-density matrix elements reflecting helicity-nonconserving amplitudes in the $t$-channel is observed.
\end{abstract}




\section{INTRODUCTION}

The study of photoproduction of light vector mesons $(\rho, \omega$, and $\phi)$ plays an important role in hadron physics to understand the nonperturbative aspect of QCD. At high energies $(W>10 \mathrm{GeV})$, Pomeron exchange in the $t$ channel describes well the photoproduction mechanism in the framework of vector-meson dominance. The Pomeron is introduced in the Regge theory for describing high-energy hadron scattering, and it is generally believed to originate from multi gluon exchange processes. At low energies near threshold $(W \sim 2$ $\mathrm{GeV}$ ), the reactions are open to the exchange of mesons and baryons, where possible "missing resonances" could be involved. In particular, the gluon dynamics dominates in the $\phi$-meson production process because the quark exchange is suppressed by the Okubo-Zweig-Iizuka (OZI) rule owing to the major $s \bar{s}$ content of the $\phi$ meson. Therefore, the photoproduction of $\phi$ mesons at low energies provides a unique opportunity to explore the behavior of Pomeron exchange and to study exotic hadronic interactions mediated by multi gluon exchanges. In fact, $\phi$-meson photoproduction from protons and deuterons has been measured in the Laser Electron Photon beamline at SPring-8 (LEPS) [1 4], the CEBAF Large Acceptance Spectrometer (CLAS) at Jefferson Lab [5-7], and the Spectrometer Arrangement for Photon induced Reactions (SAPHIR) at Bonn [8].

Spin observables are known to be a powerful tool to obtain further insights into the relevant reaction mechanisms. With the use of linearly polarized photons, the decay angular distribution of the vector meson $\phi$ can be expressed in terms of the real or imaginary part of nine spin-density matrix elements $\left(\rho_{i j}^{\alpha}\right)[9]$. These matrix elements are sensitive to the underlying reaction mechanism [10 14] . For example, the matrix element $\rho_{1-1}^{1}$ reflects the asymmetry in the contribution from natural-parity and unnatural-parity exchange [12]. There were some measurements of decay asymmetry [15] and spin-density matrix elements [16, 17] of $\phi$ production from free protons at high energies $(2.8,4.7,9.3$ and $20-40 \mathrm{GeV})$. The results were consistent with $s$-channel helicity conservation. Judging from the fact that $\rho_{1-1}^{1} \approx 0.5$, one can conclude that the $\phi$ meson is produced predominately by natural-parity Pomeron exchange at high energies.

At $E_{\gamma}=1.6-2.3 \mathrm{GeV}$, the photoproduction of $\phi$ mesons from free protons in the forward

direction [1] can be mostly described by the Pomeron and $(\pi, \eta)$ exchange in the $t$ channel. A value of 0.2 was found for $\rho_{1-1}^{1}$, which deviates from 0.5 , the limit corresponding to 
pure natural-parity exchange. It suggests a nonnegligible contribution of unnatural-parity exchange processes, in contrast to complete dominance of the Pomeron exchange at high energies.

In the measurement of coherent $\phi$ production from deuterons [2], it is reported that $\rho_{1-1}^{1}$ clearly becomes close to 0.5 . This suggests that the dominant unnatural-parity component, isovector $\pi$ exchange, is forbidden in the coupling to the isoscalar deuteron target [13, 14].

The nuclear transparency ratio of $\phi$ photoproduction for deuterons shows a large suppression of incoherent production [3] and is consistent with the $A$ dependence of the ratio observed for nuclear targets [4]. Also, $\rho_{1-1}^{1}$ was observed to be slightly larger in incoherent production from deuterons, compared with production from free protons. It coincides with destructive interference between isovector $\pi$ and isoscalar $\eta$ exchange amplitudes in the $\gamma n \rightarrow \phi n$ reaction [10, 11]. However, there is no observation of a large isospin asymmetry of $\phi$ production from nucleons. The decrease in the $\phi$-meson yields, scaled as number of nucleons in the production from deuterons, cannot be adequately explained in terms of isospin asymmetry.

A narrow bump structure was found around $E_{\gamma}=2 \mathrm{GeV}$ in $\phi$-meson production cross sections from nucleons [1] and the origin of this structure is not yet understood. Naively, it is speculated to result from the appearance of $(\pi, \eta)$ exchange near threshold. However, the measured decay angular distributions, which in principle reflect the relative weights of natural-parity and unnatural-parity processes, do not vary appreciably across the bump region. This structure cannot be described by a conventional model with the Pomeron and pseudoscalar exchange, where a monotonic energy dependence is predicted [1]. Various theoretical interpretations of this structure are proposed: the interference between unnaturalparity $\pi$ and $\eta$ exchanges [14], the interference of the isovector scalar $a_{0}$ meson with Pomeron exchange [14], the coupled-channel effect of $K \Lambda(1520)$ and $\phi N$ [18], and the existence of $N^{*}$ resonances with a large $s \bar{s}$ component [18, 19]. Nonetheless, a satisfactory description of the bump structure of $\phi$-meson photoproduction is still lacking. Comprehensive information on spin-density matrix elements will help clarify the situation.

Previously, only limited numbers of spin-density matrix elements were determined from one-dimensional decay angular distributions [1 3 , 3 , 6- 8$]$. This paper presents the results of a complete set of spin-density matrix elements in the $\gamma p \rightarrow \phi p, \gamma d \rightarrow \phi p n$, and $\gamma d \rightarrow$ $\phi d$ reactions measured in the LEPS/SPring-8 experiment. The angular distributions were 
measured via the charged-kaon decay mode of the $\phi$ meson. In Section II we describe the formulation of spin-density matrix elements and the extended maximum likelihood fit used for the determination. The experimental setup and the analysis details are introduced in Sec. [II], Sec. IV] shows the spin-density matrix elements of photoproduction of $\phi$ mesons from protons and deuterons. The interpretation of the data and discussion are given in Sec. V. Finally, Sec. VI provides a summary.

\section{SPIN-DENSITY MATRIX ELEMENTS AND EXTENDED MAXIMUM LIKE- LIHOOD}

\section{A. Decay angular distribution}

The angular distribution, $W$, of $K^{+}$decaying from $\phi$ mesons produced with a linearly polarized photon beam can be expressed as follows [9]:

$$
\begin{aligned}
W(\Omega, \Phi ; \hat{\rho})= & W(\cos \theta, \varphi, \Phi ; \hat{\rho}) \\
= & W^{0}(\cos \theta, \varphi ; \hat{\rho}) \\
& -P_{\gamma} \cos 2 \Phi W^{1}(\cos \theta, \varphi ; \hat{\rho}) \\
& -P_{\gamma} \sin 2 \Phi W^{2}(\cos \theta, \varphi ; \hat{\rho}) \\
W^{0}= & \frac{3}{4 \pi}\left[\frac{1}{2}\left(1-\rho_{00}^{0}\right)+\frac{1}{2}\left(3 \rho_{00}^{0}-1\right) \cos ^{2} \theta\right. \\
& \left.-\sqrt{2} \operatorname{Re} \rho_{10}^{0} \sin 2 \theta \cos \varphi-\rho_{1-1}^{0} \sin ^{2} \theta \cos 2 \varphi\right], \\
W^{1}= & \frac{3}{4 \pi}\left[\rho_{11}^{1} \sin ^{2} \theta+\rho_{00}^{1} \cos { }^{2} \theta\right. \\
& \left.-\sqrt{2} \operatorname{Re} \rho_{10}^{1} \sin 2 \theta \cos \varphi-\rho_{1-1}^{1} \sin ^{2} \theta \cos 2 \varphi\right], \\
W^{2}= & \frac{3}{4 \pi}\left[\sqrt{2} \operatorname{Im} \rho_{10}^{2} \sin 2 \theta \sin \varphi\right. \\
& \left.+\operatorname{Im} \rho_{1-1}^{2} \sin ^{2} \theta \sin 2 \varphi\right]
\end{aligned}
$$

Here, $\hat{\rho}$ represents the measurable parts of the nine independent spin-density matrix elements $\rho_{i j}^{\alpha}, \theta$ and $\varphi$ are the polar and azimuthal angles of decay particles in the rest frame of vector mesons, $\Phi$ is the azimuthal angle of the photon electric polarization vector with respect to the production plane of vector mesons, and $P_{\gamma}$ is the degree of linear polarization of incident photons. 
Conventionally, there are three different choices of the quantization axis $z^{\prime}$ for the decay reference system [9]: the helicity system with $z^{\prime}$ opposite to the velocity of the recoiling nucleons in the vector-meson rest frame, the Gottfried-Jackson system with $z^{\prime}$ parallel to the momentum of the photon in the vector-meson rest frame, and the Adair system with $z^{\prime}$ parallel to the photon momentum in the overall center-of-mass (CM) system.

The physical property of the production mechanism is simplest when illustrated in: the helicity system for $s$-channel helicity conservation, the Gottfried-Jackson system for t-channel helicity conservation with no absorption, and the Adair system for the spin independence in the overall CM system [20]. In addition, the Gottfried-Jackson system has the advantage that some spin-density matrix elements work as a measure of the asymmetry between processes with natural-parity and unnatural-parity exchanges in the $t$ channel [11]. In general, the values of spin-density matrix elements depend on the system chosen. Nevertheless, the difference among the three systems becomes small at very forward angles. The results in the following are presented in all three systems for easy comparison.

The contribution to the cross section from natural-parity and unnatural-parity exchanges in the $t$ channel, $\sigma^{N}$ and $\sigma^{U}$, can be determined from the density matrix elements. In the case of helicity-conserving exchanges, all spin-density matrix elements become zero, except for $\rho_{1-1}^{1}$ and $\operatorname{Im} \rho_{1-1}^{2}[12]$. That $\rho_{1-1}^{1}=-\operatorname{Im} \rho_{1-1}^{2}=+0.5(-0.5)$ corresponds to the case with pure natural-parity (unnatural-parity) exchange. The matrix element $\rho_{1-1}^{1}$ directly relates to the asymmetry in the contributions from natural-parity and unnatural-parity exchanges [12] and is expressed as

$$
\rho_{1-1}^{1}=\frac{1}{2} \frac{\sigma^{N}-\sigma^{U}}{\sigma^{N}+\sigma^{U}}
$$

In principle, nonzero values for spin-density matrix elements other than $\rho_{1-1}^{1}$ and $\operatorname{Im} \rho_{1-1}^{2}$ indicate the nonconventional OZI-evading processes such as the $s \bar{s}$ knockout [21] or nondiffractive baryon resonance production [12, 22]. Those processes are commonly expected to become important at large transferred-momentum $(|t|)$ regions for $\phi$ photoproduction.

The matrix element $\rho_{00}^{0}$ reflects the strength of the single-spin-flip exchange amplitude resulting from the components other than the Pomeron exchange. For evaluation of the relative contribution of the unnatural-parity exchange at small $|t|$, one need take into account $\rho_{00}^{0}[12]$ as follows:

$$
\frac{\sigma^{U}}{\sigma^{N}+\sigma^{U}+\sigma^{\text {spin-flip }}}=\frac{1}{2}\left(1-2 \rho_{1-1}^{1}-\rho_{00}^{0}\right) .
$$


A finite value of the spin-density matrix element $\rho_{1-1}^{0}$ comes from the amplitude responsible for the double-spin transitions where the helicity of the $\phi$ meson differs from the photon helicity by two units: $\lambda_{\gamma}= \pm 1 \rightarrow \lambda_{\phi}=\mp 1$. In many theoretical models for $\phi$-meson photoproduction, such as scalar, pseudoscalar $t$-channel exchange, and the original DonnachieLandshoff Pomeron exchange model based on the Pomeron-isoscalar photon identity, these transitions are forbidden, and $\rho_{1-1}^{0}$ is exactly zero. In the modified Donnachie-Landshoff model, $\rho_{1-1}^{0}$ in the Gottfried-Jackson system could be nonzero owing to the spin-orbital interaction inherent to the two-gluon exchange in the $t$ channel [11, 23].

\section{B. Extended maximum likelihood fit}

Experimentally we measure the angular distribution $W(\Omega, \Phi ; \hat{\rho})$ for charged kaons decaying from photoproduction events of the $\phi$ meson. A few spin-density matrix elements can be extracted from the one-dimensional angular distributions of $\cos \theta, \cos \varphi, \cos \Phi$, and

$\cos (\varphi-\Phi)[12]$. In this work, a binless maximum likelihood fit is applied to determine the nine spin-density matrix elements simultaneously.

A likelihood function with a perfect detection efficiency, $\mathcal{L}$, is defined as:

$$
\begin{aligned}
\mathcal{L} & =\prod_{i=1}^{N} P_{i} \\
& =\prod_{i=1}^{N} \frac{W\left(\Omega_{i}, \Phi_{i} ; \hat{\rho}\right)}{\int W(\Omega, \Phi ; \hat{\rho}) d \Omega d \Phi}
\end{aligned}
$$

with $P_{i}$ the normalized likelihood for each event and $W(\Omega, \Phi ; \hat{\rho})$ the probability density function. The logarithmic likelihood function $-\ln \mathcal{L}$ is minimized by the CERNLIB MINUIT package [24].

If the detector efficiency is not perfect, the probability density function is modified as

$$
P_{i}=\frac{W\left(\Omega_{i}, \Phi_{i} ; \hat{\rho}\right)}{\int W(\Omega, \Phi ; \hat{\rho}) \eta(\Omega) d \Omega d \Phi}=\frac{W\left(\Omega_{i}, \Phi_{i} ; \hat{\rho}\right)}{\mathcal{W}(\hat{\rho})} .
$$

By taking into account the efficiency $\eta(\Omega)$, the evaluation of the normalization factor $\mathcal{W}$ with a variation of $\hat{\rho}$ in the minimizing process, is technically challenging. This issue can be solved by representing the angular distribution $W(\Omega, \Phi ; \hat{\rho})$ with a set of orthogonal bases $O^{l m}[25]$ :

$$
W(\Omega, \Phi ; \hat{\rho})=\sqrt{\frac{3}{4 \pi}} \sum_{l, m} a_{l m}(\hat{\rho}) O^{l m}(\Omega, \Phi),
$$


TABLE I. Angular coefficients $a_{l m}$ and their corresponding bases $O^{l m}$.

\begin{tabular}{ccc}
\hline \hline $1, \mathrm{~m}$ & $a_{l m}$ & $O^{l m}$ \\
\hline 0,1 & $\sqrt{2 \pi} \sqrt{\frac{1}{3}}$ & $Y_{0}^{0}\left(\frac{1}{\sqrt{2 \pi}}\right)$ \\
0,2 & $\sqrt{2 \pi} \sqrt{\frac{1}{15}}\left(3 \rho_{00}^{0}-1\right)$ & $Y_{2}^{0 *}\left(\frac{1}{\sqrt{2 \pi}}\right)$ \\
0,3 & $-\sqrt{2 \pi} \sqrt{\frac{8}{5}} \operatorname{Re}\left(\rho_{10}^{0}\right)$ & $\frac{1}{\sqrt{2}}\left(-Y_{2}^{1 *}+Y_{2}^{-1 *}\right)\left(\frac{1}{\sqrt{2 \pi}}\right)$ \\
0,4 & $-\sqrt{2 \pi} \sqrt{\frac{4}{5}} \rho_{1-1}^{0}$ & $\frac{1}{\sqrt{2}}\left(Y_{2}^{2 *}+Y_{2}^{-2 *}\right)\left(\frac{1}{\sqrt{2 \pi}}\right)$ \\
$1,1-\sqrt{\pi} P_{\gamma} \sqrt{\frac{1}{3}}\left(\rho_{00}^{1}+2 \rho_{11}^{1}\right)$ & $Y_{0}^{0}\left(\frac{1}{\sqrt{\pi}} \cos (2 \Phi)\right)$ \\
1,2 & $-\sqrt{\pi} P_{\gamma} \sqrt{\frac{4}{15}}\left(\rho_{00}^{1}-\rho_{11}^{1}\right)$ & $Y_{2}^{0 *}\left(\frac{1}{\sqrt{\pi}} \cos (2 \Phi)\right)$ \\
1,3 & $\sqrt{\pi} P_{\gamma} \sqrt{\frac{8}{5}} \rho_{10}^{1}$ & $\frac{1}{\sqrt{2}}\left(-Y_{2}^{1 *}+Y_{2}^{-1 *}\right)\left(\frac{1}{\sqrt{\pi}} \cos (2 \Phi)\right)$ \\
1,4 & $\sqrt{\pi} P_{\gamma} \sqrt{\frac{4}{5}} \rho_{1-1}^{1}$ & $\frac{1}{\sqrt{2}}\left(Y_{2}^{2 *}+Y_{2}^{-2 *}\right)\left(\frac{1}{\sqrt{\pi}} \cos (2 \Phi)\right)$ \\
2,1 & $-\sqrt{\pi} P_{\gamma} \sqrt{\frac{8}{5}} \operatorname{Im}\left(\rho_{10}^{2}\right)$ & $\frac{1}{i} \frac{1}{\sqrt{2}}\left(Y_{2}^{1 *}+Y_{2}^{-1 *}\right)\left(\frac{1}{\sqrt{\pi}} \sin (2 \Phi)\right)$ \\
2,2 & $-\sqrt{\pi} P_{\gamma} \sqrt{\frac{4}{5}} \operatorname{Im}\left(\rho_{1-1}^{2}\right)$ & $\frac{1}{i} \frac{1}{\sqrt{2}}\left(Y_{2}^{2 *}-Y_{2}^{-2 *}\right)\left(\frac{1}{\sqrt{\pi}} \sin (2 \Phi)\right)$ \\
\hline
\end{tabular}

where the orthogonal conditions for each base are satisfied:

$$
\int O^{* l m} O^{l^{\prime} m^{\prime}} d \Omega d \Phi=\delta_{l l^{\prime}} \delta_{m m^{\prime}}
$$

and the coefficient for each basis $a_{l m}$ is

$$
\int O^{* l m} W(\Omega, \Phi ; \hat{\rho}) d \Omega d \Phi=\sqrt{\frac{3}{4 \pi}} a_{l m}(\hat{\rho})
$$

The explicit representations of $O^{l m}$ in terms of spherical harmonics, and the coefficients $a_{l m}$ 's are given in Table 1 .

The angular distribution of the detector efficiency $\eta(\Omega)$ can also be expanded by the same orthogonal basis $O^{l m}$ :

$$
\eta(\Omega)=\sum_{l, m} b_{l m} O^{l m}(\Omega)
$$

In a kinematic bin, the angular moment of experimental acceptance, $b_{l m}$, is evaluated as

$$
\begin{aligned}
b_{l m} & =\sqrt{\frac{3}{4 \pi}} \int O_{l m}(\Omega, \Phi) \eta(\Omega) d \Omega d \Phi \\
& =\frac{1}{N_{\text {generated }}} \sum_{i=1}^{N_{\text {accepted }}} O^{l m}\left(\Omega_{i}, \Phi_{i}\right),
\end{aligned}
$$

where the numerator and denominator sum over the accepted and isotropically generated Monte Carlo (MC) events, respectively. 
The evaluation of the normalization factor $\mathcal{W}(\hat{\rho})$ is simplified as follows:

$$
\begin{aligned}
\mathcal{W}(\hat{\rho}) & =\int W(\Omega, \Phi ; \hat{\rho}) \eta(\Omega) d \Omega d \Phi \\
& =\sum_{l, m} a_{l m}(\hat{\rho}) b_{l m} .
\end{aligned}
$$

Furthermore, the restriction that the probability distribution $P$ is normalized to 1 could be relaxed in an "extended maximum likelihood fit" [26]. The integral of the unnormalized probability density function $\mathcal{P}$ represents the total number of predicted events, $\bar{N}$, under the assumption of a Poisson variation for the measured number of events, $N$.

The extended likelihood function can be written as

$$
\begin{aligned}
\mathcal{L} & =\left(\frac{\bar{N}^{N} e^{-\bar{N}}}{N !}\right) \prod_{i=1}^{N} \mathcal{P}_{i} \\
\mathcal{P}_{i} & =\frac{\mathcal{Y} W\left(\Omega_{i}, \Phi_{i} ; \hat{\rho}\right)}{\int \mathcal{Y} W(\Omega, \Phi ; \hat{\rho}) \eta(\Omega) d \Omega d \Phi} \\
\bar{N} & =\int \mathcal{Y} W(\Omega, \Phi ; \hat{\rho}) \eta(\Omega) d \Omega d \Phi=\mathcal{Y} \mathcal{W}(\hat{\rho}),
\end{aligned}
$$

where $\mathcal{Y}$ is the actual yield. By neglecting the terms that do not depend on the fit parameters, the likelihood function to be minimized can be rewritten as follows:

$$
\begin{aligned}
-\ln \mathcal{L}(\hat{\rho}, \bar{N}) & =-\sum_{i=1}^{N} \ln \left[\mathcal{Y} I\left(\Omega_{i} ; \hat{\rho}\right)\right]+\bar{N} \\
& \left.=-\sum_{i=1}^{N} \ln \left[\bar{N} I\left(\Omega_{i} ; \hat{\rho}\right) / \mathcal{W}(\hat{\rho})\right)\right]+\bar{N}
\end{aligned}
$$

Now besides $\hat{\rho}$, the expected number of events, $\bar{N}$, becomes an additional parameter in the minimization, and it should turn out to be the number of events to be fit, $N$, in the final result. This fact could be utilized to validate the success of the minimization procedure.

\section{EXPERIMENT AND ANALYSIS}

The experiment was carried out at the LEPS/SPring-8 facility using a linearly polarized photon beam produced by backward Compton scattering of the Ar laser from 8-GeV electrons in the storage ring of SPring-8. Photons in the energy range of $1.5-2.4 \mathrm{GeV}$ were tagged by detecting recoil electrons. The photon beam with an intensity of $\sim 10^{6} / \mathrm{s}$ was directed onto liquid hydrogen or deuterium targets inside a 15-cm-long target cell. The 
direction of linear polarization was controlled vertically or horizontally by using a half-wave plate for the laser with a polarization of nearly 100\%. Charged particles emitted from the interaction points of photons with a target were detected at forward angles in the LEPS spectrometer. The spectrometer consisted of a start counter, a silica-aerogel Čerenkov counter, a silicon vertex detector, a dipole magnet, three multiwire drift chambers, and a time-offlight wall. The angular coverage of the spectrometer was about $0.4 \mathrm{rad}$ and $0.2 \mathrm{rad}$ in the horizontal and vertical directions, respectively. Particle identification was made by mass reconstruction using the measured time of flight and momentum. For more details concerning the detector configuration and the quality of particle identification, see Ref. [27]. In the present work, the integrated number of tagged photons reached at $5.6 \times 10^{12}\left(4.6 \times 10^{12}\right)$ for the hydrogen (deuterium) runs.

Events with both $K^{+}$and $K^{-}$tracks detected were selected. The spectra of a Dalitz plot of the $K^{+} K^{-} p$ final state, $K^{+} K^{-}$invariant mass, missing mass and $K^{-} p$ invariant mass for those events from hydrogen and deuterium targets are shown Fig. 1 and Fig. 2, respectively. A clear $\phi$ peak in the nominal mass of $\phi$ meson, $1.019 \mathrm{GeV}$, is seen in Fig. 11(b) and Fig. 2(b). The missing mass spectrum of the events from hydrogen shows a peak around the proton mass as seen in Fig. 1(c) while more complex structure is observed in the spectrum of the production from deuterium (see Fig. 2(c)). In the missing mass spectrum, assuming the whole deuteron as the target $\left(\mathrm{MM}_{d}(\gamma, \phi)\right)$, events of coherent $\phi$ production, $\gamma d \rightarrow \phi d$, peak at the deuteron mass of $1.875 \mathrm{GeV} / \mathrm{c}^{2}$ whereas incoherent events, $\gamma d \rightarrow \phi p n$, are distributed at relatively higher mass. This missing-mass spectrum is nicely reproduced by $\mathrm{MC}$ simulations of coherent and incoherent $\phi$ production processes. The MC simulation takes into account experimental parameters such as geometrical acceptance, energy and momentum resolutions, and the efficiency of detectors. The effects of Fermi motion, along with off-shell aspects of target nucleons inside deuterium and final-state interaction between the target and spectator nucleons, are also included to describe the $\operatorname{MM}_{d}(\gamma, \phi)$ distribution of incoherent events [2, 3]].

Furthermore, $\phi$-meson events were singled out with a cut on the invariant mass of a $K^{+} K^{-}$pair $\left|\mathrm{M}\left(K^{+} K^{-}\right)-\mathrm{M}_{\phi}\right|<0.01 \mathrm{GeV} / \mathrm{c}^{2}$, which is indicated as the region between two dashed lines in Fig. 1(b) and Fig. 2(b). For the $\gamma p \rightarrow \phi p$ events, an additional cut on the missing mass $\left|\mathrm{MM}_{\mathrm{p}}(\gamma, \phi)\right|<0.03 \mathrm{GeV} / \mathrm{c}^{2}$ is required. As seen in Figs. 1(a) and 1(d) and Figs. 2(a) and 2(d), the contamination of $\Lambda(1520)$ in the selected $\phi$-meson events is 

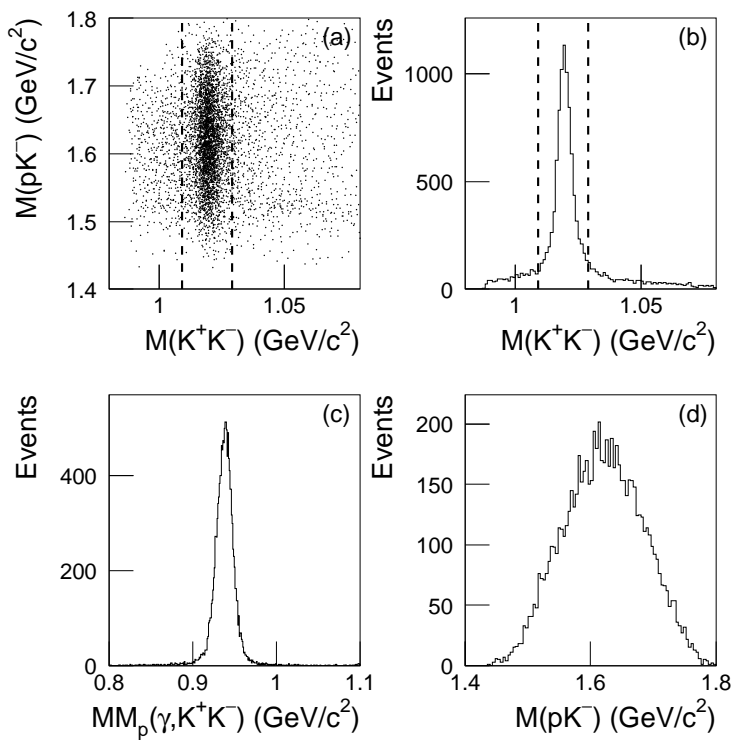

FIG. 1. Spectra for the $p\left(\gamma, K^{+} K^{-}\right) X$ reaction within the experimental acceptance: (a) Dalitz plot of $K^{+} K^{-} p$ final state, (b) the invariant mass spectrum of $K^{+} K^{-}$, (c) missing mass spectrum, and (d) the invariant mass spectrum of $K^{-} p$ for the selected $\phi$ events. The dashed lines on the invariant mass of $K^{+} K^{-}$in (a) and (b) label the region for the selection of $\phi$ events.

insignificant.

The decay angular distributions of $W(\cos \theta), W(\varphi), W(\Phi)$, and $W(\varphi-\Phi)$ in the helicity system for $\phi$ events within the experimental acceptance are shown in Fig. 3 and Fig. 4 , The polar angle distribution $W(\cos \theta)$ behaves as $\sim(3 / 4) \sin ^{2} \theta$, indicating the dominance of $s$-channel-helicity-conserving processes. That the azimuthal angle $\varphi$ of decay daughter $K^{+}$ aligns along that of the electric polarization vector $\Phi$ of the incident photon signifies a larger contribution from natural-parity exchange processes. Such a correlation between the two angles, $\varphi$ and $\Phi$, is seen to be even stronger in the production from deuterium. Quantitative information will be given in Sec. IV.

Since not all particles in the final state are detected, there is a mixture of two components, incoherent and coherent, in the selected $\phi$ events from deuterium. To disentangle the individual contributions, extra effort is needed. By following the prescription specified in Refs. [2, 3], the percentage of incoherent and coherent events, $\mathcal{R}^{1}$ and $\mathcal{R}^{2}$, are determined in the two separated missing mass regions, respectively, by a fit of the missing mass spectrum $\mathrm{MM}_{d}(\gamma, \phi)$ with the MC-simulated distributions. The division of these two regions, $\mathrm{MM}_{\mathrm{div}}$, 

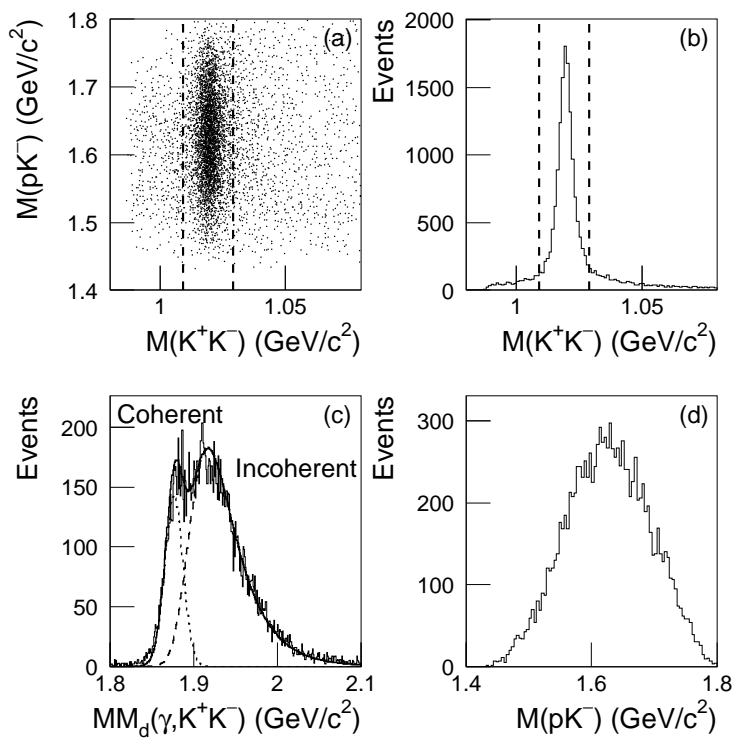

FIG. 2. Spectra for the $d\left(\gamma, K^{+} K^{-}\right) X$ reaction within the experimental acceptance: (a) Dalitz plot of $K^{+} K^{-} p$ final state, (b) the invariant mass spectrum of $K^{+} K^{-}$, (c) missing mass spectrum assuming rest deuteron target, and (d) the invariant mass spectrum of $K^{-} p$ assuming rest proton target for the selected $\phi$ events. The dashed lines on the invariant mass of $K^{+} K^{-}$in (a) and (b) label the region for the selection of $\phi$ events. The $\mathrm{MM}_{d}(\gamma, \phi)$ spectrum in (c) is fitted with the sum (solid line) of MC-simulated components of coherent (dotted line) and incoherent (dashed line) events.

is chosen to be $1.89 \mathrm{GeV} / \mathrm{c}^{2}$. Event by event, relative weights composed of either $\mathcal{R}^{1}$ or $\mathcal{R}^{2}$ are assigned to the likelihood of incoherent and coherent processes, depending on where the missing-mass of the event sits. In contrast to Eq. (21), the likelihood function for each event is represented as the weighted sum of the individual likelihoods from the incoherent and coherent processes:

$$
\begin{aligned}
& -\ln \mathcal{L}\left(\hat{\rho}^{\mathrm{inco}}, \hat{\rho}^{\mathrm{co}}, \bar{N}\right)= \\
& -\sum_{i=1}^{N} \ln \left\{\bar{N}\left[\mathcal{R}_{i} \frac{W\left(\Omega_{i} ; \hat{\rho}^{\mathrm{inco}}\right)}{\mathcal{W}\left(\hat{\rho}^{\mathrm{inco}}\right)}+\left(1-\mathcal{R}_{i}\right) \frac{W\left(\Omega_{i} ; \hat{\rho}^{\mathrm{co}}\right)}{\mathcal{W}\left(\hat{\rho}^{\mathrm{co}}\right)}\right]\right\}+\bar{N} .
\end{aligned}
$$

Here $\mathcal{R}_{i}$ is either $\mathcal{R}^{1}$ or $\mathcal{R}^{2}$ according to the associated missing-mass value of the event; the spin-density matrix elements for the incoherent reaction $\hat{\rho}^{\text {inco }}$ and coherent reaction $\hat{\rho}^{\text {co }}$, together with the expected number of events, $\bar{N}$, are the parameters to be determined in the extended maximum likelihood fit. 

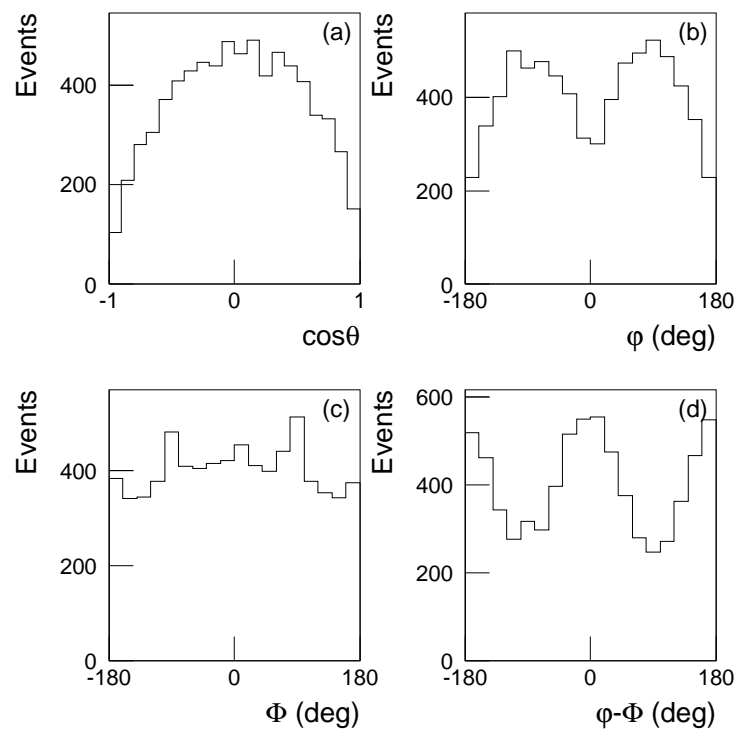

FIG. 3. Decay angular distributions for the $p\left(\gamma, K^{+} K^{-}\right) X$ reaction in the helicity system within the experimental acceptance: (a) $W(\cos \theta),(\mathrm{b}) W(\varphi),(\mathrm{c}) W(\Phi)$, and (d) $W(\varphi-\Phi)$.

The extended maximum likelihood fit was performed in the framework of the MINUIT package. To locate the global minimum, the initial values of fit parameters were chosen to be the converged results from many test fits where a random point in the allowed range of the multidimensional parameter space was used as the start for the minimization. After a call of minimization by the method of MIGRAD, the statistic error estimation was done by a MINOS error analysis. The returned symmetric parabolic error was reported.

Several procedures were checked to ensure the fit quality. At first, the fit status returned by MINUIT was required to have a normal convergence. The global correlation coefficient of each parameter should be greater than zero and less than 0.99 , to avoid a wrong estimation of the statistic error for the case of uncorrelated or strongly correlated parameters. The typical value of the global correlation coefficient was distributed between 0.2 and 0.6 . Second, the fit parameter for the number of events must be consistent with the input statistics, an advantage from the use of an extended maximum likelihood fit. Finally, reasonable agreement between the one-dimensional angular distributions of $\cos \theta, \varphi, \Phi$, and $\varphi-\Phi$ from the input events and the fitted values of $\hat{\rho}$, was checked. The $\chi^{2}$ value per degree of freedom from a normalization fit is required to be in the range of 1.0-3.0. In general, a fit for a given kinematic bin associated with small statistics was likely to fail in this quality check. 

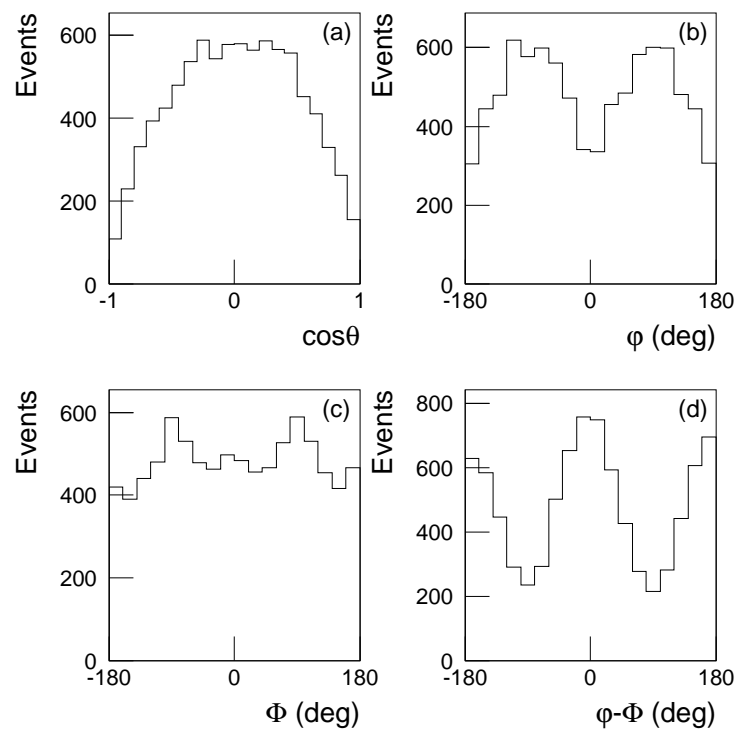

FIG. 4. Decay angular distributions for the $d\left(\gamma, K^{+} K^{-}\right) X$ reaction in the helicity system within the experimental acceptance: (a) $W(\cos \theta),(\mathrm{b}) W(\varphi)$, (c) $W(\Phi)$, and (d) $W(\varphi-\Phi)$.

The spin-density matrix elements do not depend on the beam normalization which is typically the main systematic uncertainty of cross-section measurements at LEPS. We evaluated the systematic uncertainty in the following way. Ensembles of MC events were generated with specific sets of spin-density matrix elements being the same as those found in the real data. Those MC events were then filtered by the experimental detection efficiency. Subsequently, those events with statistics similar to the real data were analyzed for the determination of spin-density matrix elements $\hat{\rho}$. The mean of the distribution of obtained $\hat{\rho}$ for many trials, the so-called pull distribution, was determined. The deviation of the mean from the generated value of $\hat{\rho}$ for $\mathrm{MC}$ events contributed to the estimation of the systematic uncertainty.

Another source came from the background reactions [e.g., nonresonant $K^{+} K^{-}$and $\Lambda(1520)$ production]. This bias was estimated by comparing the results in the signal region $\left[\left|\mathrm{M}\left(K^{+} K^{-}\right)-\mathrm{M}_{\phi}\right|<0.01 \mathrm{GeV} / \mathrm{c}^{2}\right]$ and the sideband region $\left[0.01<\left|\mathrm{M}\left(K^{+} K^{-}\right)-\mathrm{M}_{\phi}\right|<0.02\right.$ $\left.\mathrm{GeV} / \mathrm{c}^{2}\right]$, by taking into account different signal-to-background ratios in these two regions . In general, the signal-to-background ratio in the defined $\phi$-event region is good enough as shown in Figs. 1(a) and 1(b) and Figs. 2(a) and 2(b). Thus this bias was found to be small (of the order of 0.01-0.05 except at regions of larger $|t|$ ). Since the statistics in the sideband 
region was not always enough for a reliable fit, we conservatively included this bias in the systematic error, rather than applying the corresponding correction to the results.

For the $\phi$-meson production from deuterium, there are additional sources of systematic error: the off-shell effect for the incoherent process in the MC simulation and the division point in the missing mass regions for disentanglement [2, 3]. The choice of the division point $\mathrm{MM}_{\text {div }}$ would affect the contents of coherent and incoherent events on the two separated regions. Four choices of $\mathrm{MM}_{\text {div }}, 1.875,1.88,1.89$ and $1.90 \mathrm{GeV} / \mathrm{c}^{2}$, were used for evaluating the systematic uncertainty.

\section{RESULTS}

We measured the spin-density matrix elements of $\phi$-meson photoproduction in the region of $1.57<E_{\gamma}<2.37 \mathrm{GeV}$ and $|\tilde{t}|<0.2 \mathrm{GeV}^{2} / \mathrm{c}^{2}$. We define $\tilde{t}$ as $t-t_{\text {min }}^{p}$ or $t-t_{\text {min }}^{d}$, which is the squared four-momentum transfer $t$ subtracted by its minimum value for the corresponding photon energy bin, under the assumption that a proton or deuteron is at rest. The binning sizes were $0.2 \mathrm{GeV}$ for $E_{\gamma}$ and $0.05 \mathrm{GeV}^{2} / \mathrm{c}^{2}$ for $\tilde{t}$. The real or imaginary parts of spin-density matrix elements $\rho_{i j}^{\alpha}$ for the $\gamma p \rightarrow \phi p, \gamma d \rightarrow \phi p n$, and $\gamma d \rightarrow \phi d$ reactions were determined by the method as described in Secs. [I] and [II.

\section{A. Production from free protons: $\gamma p \rightarrow \phi p$}

The spin-density matrix elements of the $\gamma p \rightarrow \phi p$ reaction in the helicity, GottfriedJackson, and Adair systems are shown in Figs. 5, 6] and 17, respectively. Within the error there is no strong energy dependence in the measured region of $1.77<E_{\gamma}<2.37 \mathrm{GeV}$. Clear nonzero values for $\rho_{1-1}^{1}$ and $\operatorname{Im} \rho_{1-1}^{2}$ are seen in all three systems, and $\rho_{1-1}^{1} \approx 0.2$. According to Eq. (5), a sizable 30\% contribution of unnatural-parity exchange processes, other than the the natural-parity Pomeron exchange, is observed.

In the helicity system, small but nonzero positive values for $\rho_{00}^{0}$ and negative ones for $\operatorname{Re} \rho_{10}^{0}$ are observed. The $\rho_{00}^{0}\left(\operatorname{Re} \rho_{10}^{0}\right)$ are of positive (negative) values. The finiteness of $\rho_{00}^{0}$ indicates the presence of amplitudes violating $s$-channel-helicity in the $\phi$ production.

In the Gottfried-Jackson system, $\rho_{00}^{0}$ seems to be larger and $\operatorname{Re} \rho_{10}^{0}$ turns to be positive, compared to the results in the helicity system. The increase in the magnitude of $\rho_{00}^{0}$ suggests 


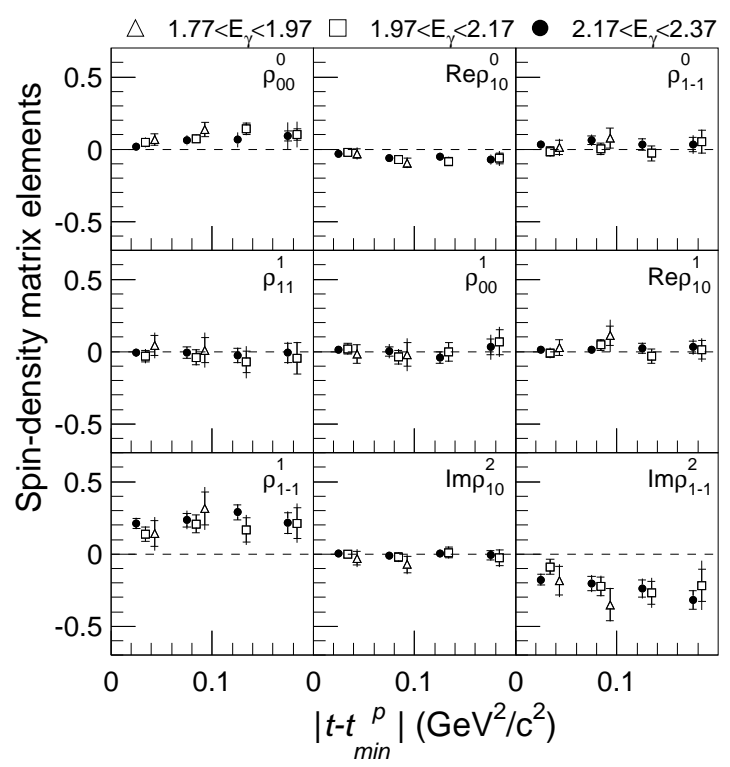

FIG. 5. Spin-density matrix elements for the $\gamma p \rightarrow \phi p$ reaction in the helicity system as a function of $\left|t-t_{\text {min }}^{p}\right|$ in various $E_{\gamma}$ regions. The vertical bars are for statistical error only.

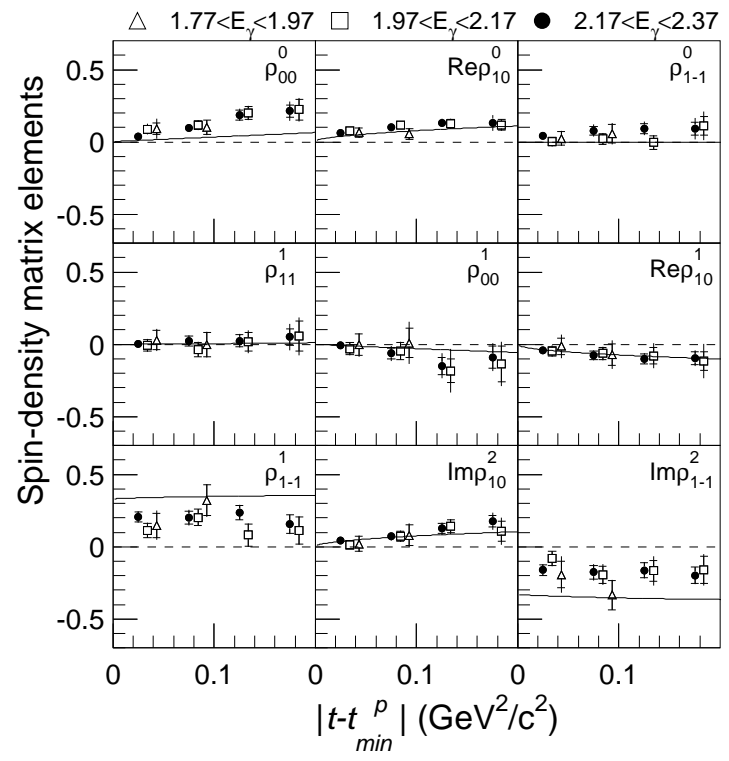

FIG. 6. Spin-density matrix elements for the $\gamma p \rightarrow \phi p$ reaction in the Gottfried-Jackson system as a function of $\left|t-t_{\text {min }}^{p}\right|$ in various $E_{\gamma}$ regions. The vertical bars are for statistical error only. The solid lines are the theoretical predictions of $\hat{\rho}$ for the $\gamma p \rightarrow \phi p$ reaction at $E_{\gamma}=2 \mathrm{GeV}$ [11]. 


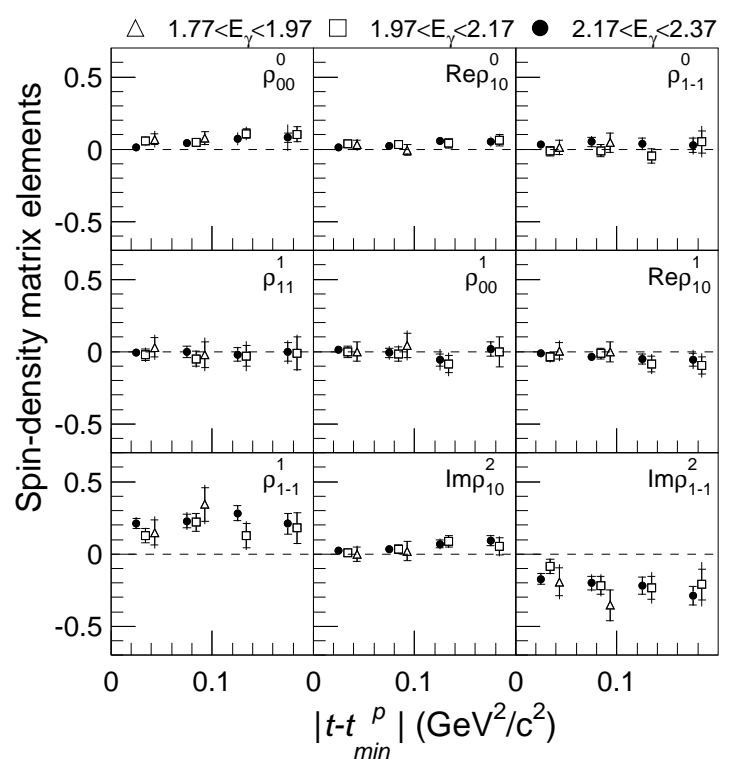

FIG. 7. Spin-density matrix elements for the $\gamma p \rightarrow \phi p$ reaction in the Adair system as a function of $\left|t-t_{\text {min }}^{p}\right|$ in various $E_{\gamma}$ regions. The vertical bars are for statistical error only.

a stronger violation of helicity conservation in the $t$ channel. Specifically, those spin-density matrix elements reflecting helicity-nonconserving amplitudes such as $\rho_{00}^{0}, \rho_{1-1}^{0}, \rho_{00}^{1}, \operatorname{Re} \rho_{10}^{1}$, and $\operatorname{Im} \rho_{10}^{2}$ become clearly nonzero at large $\tilde{t}$. Figure 6 shows a comparison with the prediction from a model based on the dominance of the Donnachie-Landshoff Pomeron plus $(\pi, \eta)$ exchange channels [11] at $E_{\gamma}=2 \mathrm{GeV}$. This model correctly predicts the sign of each matrix element. However, the $t$ dependence for $\rho_{00}^{0}, \rho_{1-1}^{0}$, and $\rho_{00}^{1}$ is not correctly predicted. Also the absolute scale of $\rho_{1-1}^{1}$ and $\operatorname{Im} \rho_{1-1}^{2}$ are overpredicted, which means that the contribution from the unnatural-parity exchange processes is not sufficient in this model.

The $\hat{\rho}$ values in the Adair system are rather similar to those in the helicity system except for an opposite sign for $\operatorname{Re} \rho_{10}^{0}$ and $\operatorname{Im} \rho_{10}^{2}$.

\section{B. Incoherent production from deuterons: $\gamma d \rightarrow \phi p n$}

The spin-density matrix elements for the incoherent production from deuterons in three angular systems are shown in Figs. 8, 9] and 10, respectively. The energy dependence is also insignificant. Because of statistical constraint, the measured range of $\tilde{t}$ is limited to $|\tilde{t}|<0.15$. In general, the results are quite similar to those of the production from free 


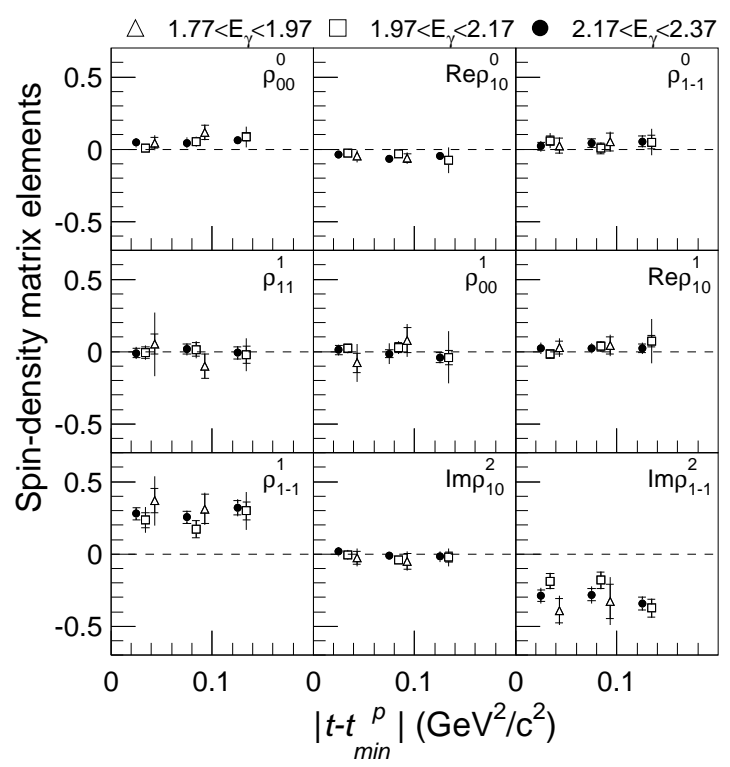

FIG. 8. Spin-density matrix elements for the $\gamma d \rightarrow \phi p n$ reaction in the helicity system as a function of $E_{\gamma}$ and $\left|t-t_{m i n}^{p}\right|$. The vertical bars are for statistical error only.

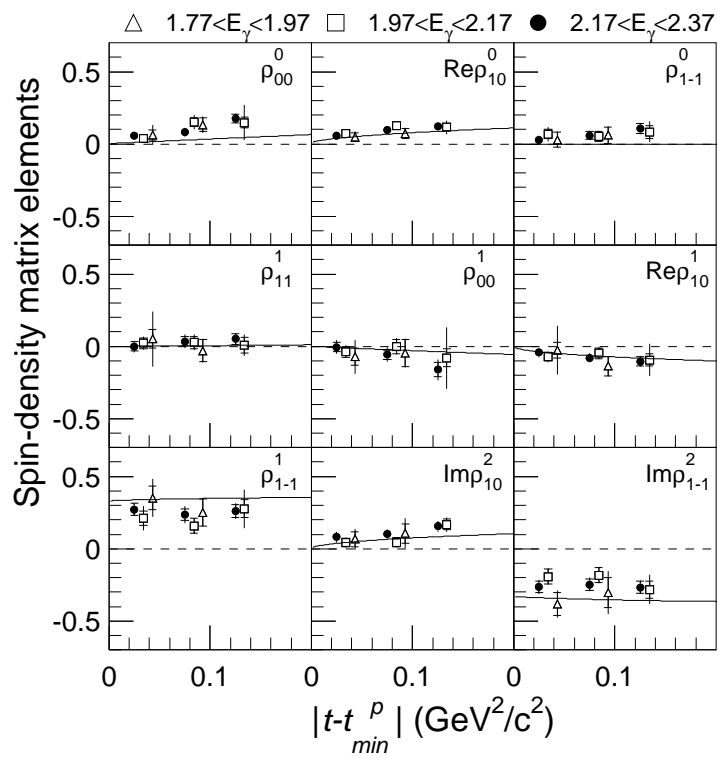

FIG. 9. Spin-density matrix elements for the $\gamma d \rightarrow \phi p n$ reaction in the Gottfried-Jackson system as a function of $E_{\gamma}$ and $\left|t-t_{\text {min }}^{p}\right|$. The vertical bars are for statistical error only. The solid lines are the theoretical predictions of $\hat{\rho}$ for the $\gamma p \rightarrow \phi p$ reaction at $E_{\gamma}=2 \mathrm{GeV}[11]$. 


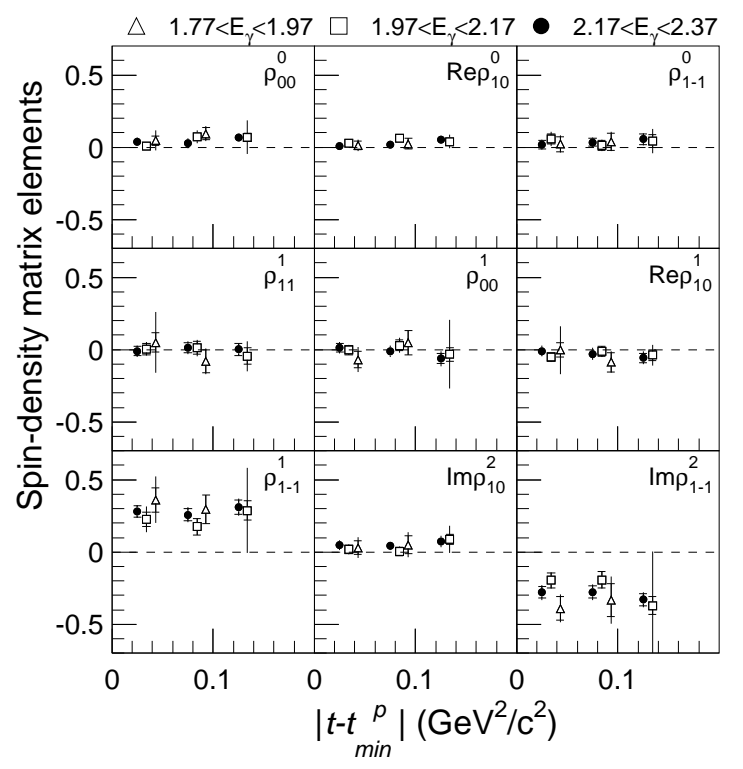

FIG. 10. Spin-density matrix elements for the $\gamma d \rightarrow \phi p n$ reaction in the Adair system as a function of $E_{\gamma}$ and $\left|t-t_{m i n}^{p}\right|$. The vertical bars are for statistical error only.

protons except that the absolute values of $\rho_{1-1}^{1}$ and $\operatorname{Im} \rho_{1-1}^{2}$ are slightly larger $\left(\rho_{1-1}^{1} \approx 0.25\right)$. This suggests that the contribution from unnatural-parity exchange processes is reduced in the production from neutrons. It could be interpreted as a destructive interference effect among the unnatural-parity $(\pi, \eta)$-exchange processes in the $\phi$-meson production from the neutron [10, 11].

\section{Coherent production from deuterons: $\gamma d \rightarrow \phi d$}

Figures 11, 12 and 13 display the spin-density matrix elements for the coherent production from deuterium in three angular systems. A distinct feature is a strong increase of the absolute values of $\rho_{1-1}^{1}$ and $\operatorname{Im} \rho_{1-1}^{2}$, and $\rho_{1-1}^{1} \approx 0.45$, compared to what is observed in the reactions of $\gamma p \rightarrow \phi p$ and $\gamma d \rightarrow \phi p n$. This again suggests that the contribution from the unnatural-parity exchange processes is significantly reduced. It could be understood as a result of the forbidden coupling of the isovector $\pi$ exchange with the isoscalar deuteron target. All the other components in $\hat{\rho}$ are similar to those in the production from nucleons. In the helicity system the smallness of $\rho_{00}^{0}$ (less than 10\%) in the small- $|t|$ region is the same as what was observed in the region of large $|t|$ by CLAS $[6]$. 


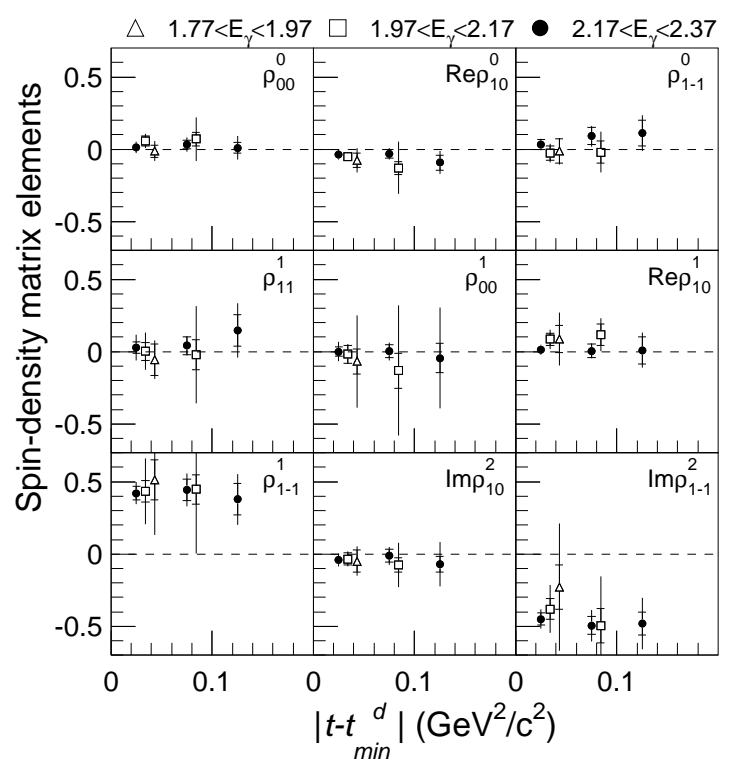

FIG. 11. Spin-density matrix elements for the $\gamma d \rightarrow \phi d$ reaction in the helicity system as a function of $E_{\gamma}$ and $\left|t-t_{\text {min }}^{d}\right|$. The vertical bars are for statistical error only.

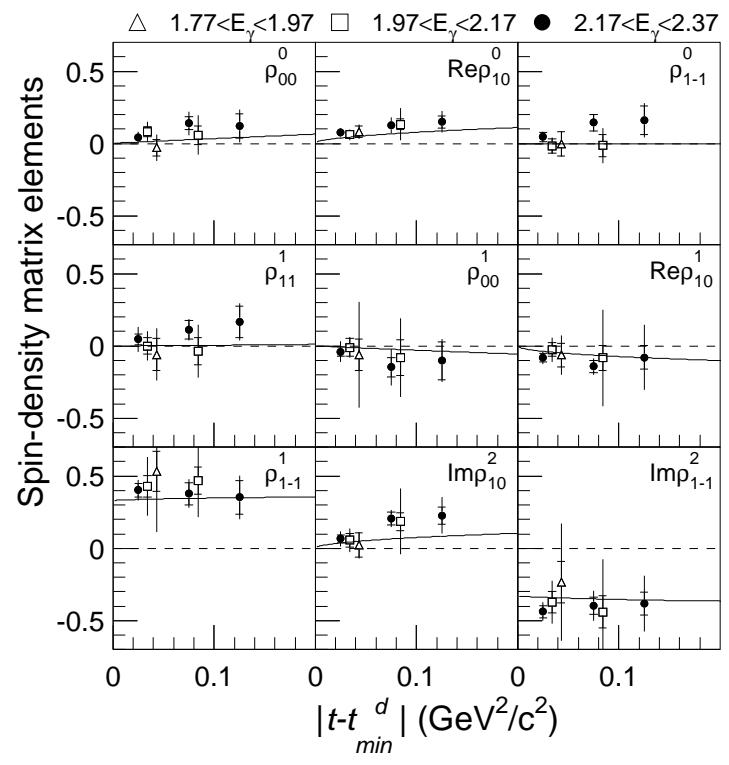

FIG. 12. Spin-density matrix elements for the $\gamma d \rightarrow \phi d$ reaction in the Gottfried-Jackson system as a function of $E_{\gamma}$ and $\left|t-t_{\text {min }}^{d}\right|$. The vertical bars are for statistical error only. The solid lines are the theoretical predictions of $\hat{\rho}$ for the $\gamma p \rightarrow \phi p$ reaction at $E_{\gamma}=2 \mathrm{GeV}[11]$. 


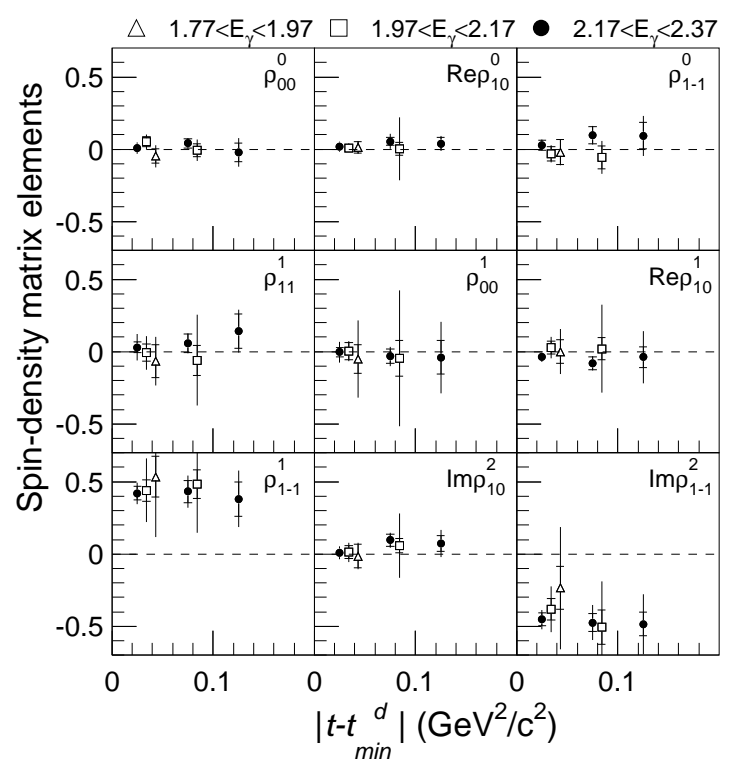

FIG. 13. Spin-density matrix elements for the $\gamma d \rightarrow \phi d$ reaction in the Adair system as a function of $E_{\gamma}$ and $\left|t-t_{\text {min }}^{d}\right|$. The vertical bars are for statistical error only.

\section{DISCUSSION}

The finiteness of the spin-density matrix elements $\rho_{00}^{0}, \rho_{1-1}^{0}, \rho_{00}^{1}, \operatorname{Re} \rho_{10}^{1}$, and $\operatorname{Im} \rho_{10}^{2}$ is clearly observed in the Gottfried-Jackson system. This suggests the presence of helicitynonconserving effects in the $t$-channel exchange processes for $\phi$-meson photoproduction, (e.g., two-gluon exchange as mentioned before [23]). Relatively, the helicity-nonconserving effects become less in the helicity ( $s$-channel) and Adair systems.

Previously, we reported a nonzero value of $\rho_{1-1}^{0}(0.12 \pm 0.03)$ for the $\gamma p \rightarrow \phi p$ reaction in the region of $\left|t-t_{\text {min }}^{p}\right|<0.2 \mathrm{GeV}^{2} / \mathrm{c}^{2}$ at $E_{\gamma}=1.77-1.97 \mathrm{GeV}[1]$, while $\rho_{1-1}^{0}$ became less (about $0.04 \pm 0.02$ ) at $E_{\gamma}=1.97-2.17 \mathrm{GeV}$. The measurement was done with a fit on the one-dimensional azimuthal angle distribution $W(\varphi)$ in the Gottfried-Jackson system. In Refs. [13, 14], the authors argued that $\rho_{1-1}^{0}$, which could only come from a spin-orbit interaction, must be close to zero near threshold and should monotonically increase with photon energy. However, the results were obtained in the region of $\left|t-t_{\min }^{p}\right|<0.2 \mathrm{GeV}^{2} / \mathrm{c}^{2}$, instead of at $t=t_{\text {min }}^{p}$. The effect of $\tilde{t}$ dependence shown in Fig. 6] should be taken into account.

Using the current data set from the hydrogen runs, we repeated the same analysis. The 
results are shown in Fig. 14. Figures 14(a) and 14(b) display the $W(\varphi)$ distributions and the fitted $\rho_{1-1}^{0}$ for the $\gamma p \rightarrow \phi p$ reaction in the regions of $\left|t-t_{m i n}^{p}\right|<0.05 \mathrm{GeV}^{2} / \mathrm{c}^{2}$ and $\left|t-t_{\text {min }}^{p}\right|<0.2 \mathrm{GeV}^{2} / \mathrm{c}^{2}$ at three $E_{\gamma}$ bins. Obviously there is some $t$ dependence. The current measurement at $\left|t-t_{\text {min }}^{p}\right|<0.2 \mathrm{GeV}^{2} / \mathrm{c}^{2}$ is consistent with the previous results. At the smallest $|\tilde{t}|$ bin, $\rho_{1-1}^{0}$ is about 0.05 , which is consistent with the results shown in Fig. 6. Within the error, we could not draw any conclusion on the monotonic increase of $\rho_{1-1}^{0}$ with the photon energy but at least there is no large jump in the energy dependence. It is interesting to note that the $\rho_{1-1}^{0}$ value at $E_{\gamma}=1.97-2.17 \mathrm{GeV}$, where the peak of the bump structure in the $\phi$-meson production cross section appears, seems systematically smaller than those in the neighboring energy bins.

The measurements of the diffractive $\phi$ production at low energies are qualitatively consistent with the description of natural-parity Pomeron and unnatural-parity pseudoscalar exchanges in the $t$ channel [12]. The latter term includes the $(\phi, \eta)$ exchange and accounts for about $30 \%$ of the production cross section in the $\gamma p \rightarrow \phi p$ reaction. In the coherent production process, the isovector $\pi$ exchange is eliminated, and the natural-parity exchange becomes quite dominant. This observation reflects the fact that the $\eta$-exchange channel is relatively small. A slight increase in the contribution for natural-parity exchange is seen in the incoherent production, being consistent with the theoretical prediction, if one takes into account the destructive effect of $\pi$ - and $\eta$-exchanges in the $\gamma n \rightarrow \phi n$ reaction [10, 11].

A bump structure in the differential cross section at forward angles is observed at $E_{\gamma}=2$ $\mathrm{GeV}$ in both the production from free protons and the incoherent production from deuterons. The origin of this structure should be common for production from both protons and neutrons. Thus it is unlikely that the interference effect from the $(\pi, \eta)$ exchange is responsible [14]. Judging from the smallness of $\hat{\rho}$ except $\rho_{1-1}^{1}$ and $\operatorname{Im} \rho_{1-1}^{2}$ in the helicity frame, we see that the helicity-nonconserving amplitudes is rather limited for the $s$ channel in the very forward direction. There is also no significant variation in the spin-density matrix elements across the bump region. Therefore we conclude that this bump structure is also unlikely to be caused by any nucleon resonance states containing large $s \bar{s}$ content [18, 19] unless there are some complicated interference effects. 

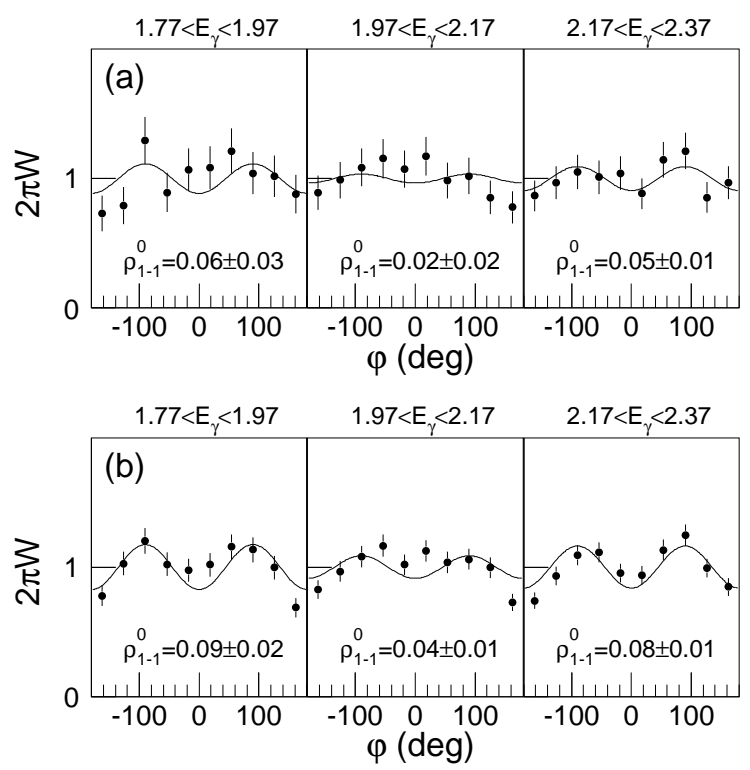

FIG. 14. (a) Azimuthal angle distribution $W(\varphi)$ for $\gamma p \rightarrow \phi p$ at $\left|t-t_{\text {min }}^{p}\right|<0.05 \mathrm{GeV}^{2} / \mathrm{c}^{2}$ in the Gottfried-Jackson system. The solid curves are the fit to the data. (b) Same as (a) but at at $\left|t-t_{\min }^{p}\right|<0.2 \mathrm{GeV}^{2} / \mathrm{c}^{2}$.

\section{SUMMARY}

In summary, we presented the measurement of decay angular distributions of $\phi$ mesons produced from protons and deuterons with linearly polarized photons at very forward angles at $E_{\gamma}=1.77-2.37 \mathrm{GeV}$. Nine spin-density matrix elements representing the angular distributions in the $K^{+} K^{-}$decay mode are obtained simultaneously by the extended maximum likelihood fit in three different coordinate frames. There is no strong energy dependence observed in the measured energy region, although some $t$ dependence is found. Small but finite helicity-nonconserving effects are seen in the $t$ channel but they become less significant in the $s$ channel.

Based on the measurement of $\rho_{1-1}^{1}$ and $\operatorname{Im} \rho_{10}^{2}$, unnatural-parity exchange processes are no longer negligible near threshold, even though the natural-parity Pomeron exchange process still dominates. The unnatural-parity processes are significantly reduced in the coherent production from deuterium. This fact can be interpreted to appear as the result of the suppression of isovector $\pi$ exchange. The averaged contribution of the unnatural-parity processes in incoherent production decreases slightly and could be due to the destructive 
interference of the $(\pi, \eta)$-exchange processes in production from neutrons.

A theoretical model based on the dominance of the Donnachie-Landshoff Pomeron plus

$(\pi, \eta)$-exchange channels [11] gives a reasonable prediction at $E_{\gamma}=2 \mathrm{GeV}$ but obviously there is room for quantitative improvement. New and comprehensive information on spin observables available from this work should help to differentiate the theoretical models. Hopefully, this will lead to a better picture of the $\phi$-meson photoproduction at low energies, especially to shed light on whether any exotic channel is necessary to account for the bump structure at $E_{\gamma} \sim 2 \mathrm{GeV}$.

A manifestation of an unnatural-parity exchange component in the photoproduction of $\phi$ mesons near threshold regions was already observed. New programs at LEPS and CLAS aim to perform measurements at $E_{\gamma}=2.5-3.5 \mathrm{GeV}$, and to study double polarization observables with polarized proton and deuterium targets. Such experimental efforts shall bring further understanding of the appearance of bump structure in the $\phi$-meson photoproduction near threshold.

\section{ACKNOWLEDGMENTS}

The authors thank the SPring- 8 staff for their great help during the operation of the LEPS experiment. This research was supported in part by the Ministry of Education, Science, Sports and Culture of Japan, the National Science Council of Republic of China (Taiwan), Korea Research Foundation Grant No. 2009-0089525, and National Science Foundation Grant No. PHY-0653454.

[1] T. Mibe et al. (LEPS Collaboration), Phys. Rev. Lett. 95, 182001 (2005).

[2] W.C. Chang et al. (LEPS Collaboration), Phys. Lett. B 658, 209 (2008).

[3] W.C. Chang et al. (LEPS Collaboration), Phys. Lett. B 684, 6 (2010).

[4] T. Ishikawa et al. (LEPS Collaboration), Phys. Lett. B 608, 215 (2005)

[5] E. Anciant et al. (CLAS Collaboration), Phys. Rev. Lett. 85, 4682 (2000).

[6] T. Mibe et al. (CLAS Collaboration), Phys. Rev. C 76, 052202 (2007).

[7] X. Qian et al. (CLAS Collaboration), Phys. Lett. B 680, 417 (2009).

[8] J. Barth et al. (SAPHIR Collaboration), Eur. Phys. J. A 17, 269 (2003). 
[9] K. Schilling, P. Seyboth, and G. Wolf, Nucl. Phys. B15, 397 (1970).

[10] A.I. Titov, T.S.-H. Lee, and H. Toki, Phys. Rev. C 59, R2993 (1999).

[11] A.I. Titov, T.S.-H. Lee, H. Toki, and O. Streltsova, Phys. Rev. C 60, 035205 (1999).

[12] A.I. Titov and T.S.-H. Lee, Phys. Rev. C 67, 065205 (2003).

[13] A. I. Titov, M. Fujiwara, and T. S.-H. Lee Phys. Rev. C 66, 022202 (2002).

[14] A.I. Titov and B. Kämpfer, Phys. Rev. C 76, 035202 (2007).

[15] H. J. Halpern et al., Phys. Rev. Lett. 29, 1425 (1972).

[16] J. Ballam et al., Phys. Rev. D 7, 3150 (1973).

[17] M. Atkinson et al., Z. Phys. C 27, 233 (1985).

[18] S. Ozaki, A. Hosaka, H. Nagahiro, and O. Scholten, Phys. Rev. C 80, 035201 (2009); 81, 059901(E) (2010).

[19] A. Kiswandhi, J.J. Xie, and S.N. Yang, Phys. Lett. B 691, 214 (2010).

[20] J. Ballam et al., Phys. Rev. D 5, 545 (1972).

[21] A.I. Titov, Y. Oh, S.N. Yang, and T. Morii, Phys. Rev. C 58, 2429 (1998)

[22] Q. Zhao, B. Saghai, and J. S. Al-Khalili, Phys. Lett. B 509, 231 (2001).

[23] J.-M. Laget, Phys. Lett. B 489, 313 (2000).

[24] F. James, CERN Applications Software Group, CERN Program Library Long Writeup D506, 1998.

[25] S. U. Chung et al., Phys. Rev. Lett. 40, 355 (1978); S. U. Chung, Phys. Rev. D 56, 7299 (1997).

[26] L. Lyons, W. M. Allison, and J. P. Comellas, Nucl. Instrum. Methods Phys. Res., Sect. A 245, 530 (1986); R. Barlow, ibid., Sect. A 297, 496 (1990).

[27] M. Sumihama et al. (LEPS Collaboration), Phys. Rev. C 73, 035214 (2006). 